\title{
Analytical solution of coupled non-linear second order reaction differential equations in enzyme kinetics
}

\author{
Govindhan Varadharajan, Lakshmanan Rajendran
}

Department of Mathematics, The Madura College, Madurai, India; raj_sms@rediffmail.com

Received 16 April 2011; revised 10 May 2011; accepted 17 May 2011.

\begin{abstract}
The coupled system of non-linear second-order reaction differential equation in basic enzyme reaction is formulated and closed analytical expressions for substrate and product concentrations are presented. Approximate analytical method (He's Homotopy perturbation method) is used to solve the coupled non-linear differential equations containing a non-linear term related to enzymatic reaction. Closed analytical expressions for substrate concentration, enzyme substrate concentration and product concentration have been derived in terms of dimensionless reaction diffusion parameters $k, \lambda$ and $\varepsilon$ using perturbation method. These results are compared with simulation results and are found to be in good agreement. The obtained results are valid for the whole solution domain.
\end{abstract}

Keywords: Non-Linear Reaction Equations; Mathematical Modelling; Steady-State; Homotopy Perturbation Method; Simulation

\section{INTRODUCTION}

Enzyme kinetics is the study of the chemical reaction that are catalysed by enzymes. In enzyme kinetics, the reaction rate is measured and the effects of varying the conditions of the reaction investigated. Enzymes are usually protein molecules that manipulate other molecules the enzymes substrate. These target molecules bind to an enzyme's active site and are transformed into products through a series of steps known as the enzymatic mechanism. These mechanisms can be divided into single-substrate and multiple-substrate mechanisms. To understand the role of enzyme kinetics, the researcher has to study the rates of reaction, the temporal behaviours of the various reactants and the conditions which influence the enzyme kinetics. Introduction with a mathematical bent is given in the books by Rubinow [1], Murray [2], Segel [3] and Roberts [4].
The generalized theoretical treatment of the transient-state kinetics of enzyme reaction system described [5-9] under the conditions $\left[E_{0}\right] \gg\left[S_{0}\right]$, the enzyme concentration $[E]$ remains effectively constant during the course of the reaction and only the substrate concentration $[S]$ changes appreciably with time. The rate of second-order reactions in chemistry are frequeuntly studied within PFO kinetics [10,11]. Numerical studies of reaction (1) far from the QSS or equilibrium approximations demonstrate that if the excess reactant concentration ratio $\left[E_{0}\right]:\left[S_{0}\right]$ say, is less than 10 -fold, appreciable errors are introduced in the pseudo-first-order kinetics description [7]. Silicio and Peterson [10] have numerical estimates for second-order reactions that show that the fractional error in the observed pseudo-firstorder constant is less than $10 \%$ if the reactants ratio is 10-fold. However, Corbett [12] has found that a pseudo-first-order reaction can yield more accurate data than is generally realized, even if only a two-fold excess of one the reactants is employed. For enzyme catalyzed reactions, Kasserra and Laidler [5] suggest that an excess of initial enzyme concentration is necessary to guarantee that the reaction follows first-order kinectics in transient-phase studies.

Schnell and Maini [13] have shown that, under the condition $\left[E_{0}\right] \gg>\left[S_{0}\right]$, the appropriate frame work to study the Michaelis-Menten reaction (1) is the reverse quasi-steady-state approximation (rQSSA) or equilibrium approximation. The reverse quasi-steady-state assumption considers the substrate $S$ to be in a quasisteady-state with respect to the enzyme-substrate complex $C$ by assuming that $\frac{d[S]}{d t} \approx 0$. Recently Schnell and Mendoza [14] have obtained the analytical expression of concentration by the linearization of the Michaelis-Menten reaction by pseudo-first-order kinetics. Recently Meena, Eswari and Rajendran [15] have derived the analytical solution of non-linear reaction equations containing a non-linear term related to the enzymatic reaction using variational iteration method (VIM).

The purpose of this communication is to derive closed 
analytical expressions for substrate concentration, enzyme substrate concentration and product concentration interms of dimensionless reaction diffusion parameters $k$, $\lambda$ and $\varepsilon$ using Homotopy perturbation method (HPM) and compartive study of the same with Numerical simulation.

\section{MATHEMATICAL FORMULATION OF THE PROBLEM}

The model of an enzyme action, initially developed by Michaelis and Menten revealed the binding of free enzyme to the reactant forming an enzyme-reactant complex. In turn this complex undergoes a transformation, releasing the product and free enzyme and the presence of free enzyme leads to another round of binding to a new reactant. The simplest reversible association between an enzyme $E$ and a substrate $S$ yield an intermediate enzyme-substrate complex $C$ that irreversibly breaks down to form a product $P$, and the mechanism is often written as:

$$
E+S \stackrel{k_{1}}{\underset{k_{-1}}{\longrightarrow}} C \stackrel{k_{2}}{\longrightarrow} E+P
$$

This mechanism illustrates the binding of substrate $\mathrm{S}$ and release of product $P . E$ is the free enzyme and $C$ is the enzyme-substrate complex. The time evolution of reaction (1) is obtained by applying the law of mass action to yield the set of coupled non-linear differential equation [14]:

$$
\begin{aligned}
& \frac{d[S]}{d t}=k_{1}\left[-\left(\left[E_{0}\right]-[C][S]\right)+K_{S}[C]\right] \\
& \frac{d[C]}{d t}=k_{1}\left[\left(\left[E_{0}\right]-[C][S]\right)+K_{M}[C]\right] \\
& \frac{d[P]}{d t}=k_{2}[C]
\end{aligned}
$$

and by imposing the laws of mass action:

$$
\begin{aligned}
& {\left[E_{0}\right]=[E](t)+[C](t)} \\
& {\left[S_{0}\right]=[S](t)+[C](t)+[P](t)}
\end{aligned}
$$

with initial conditions at $t=0$

$$
[S]=\left[S_{0}\right],[E]=\left[E_{0}\right],[C]=0,[P]=0
$$

In this system the parameters $k_{1}, k_{-1}$ and $k_{2}$ are positive rate constants, $K_{S}=k_{-1} / k_{1}$ is the equilibrium dissociation constant, $K=k_{2} / k_{1}$ the Van Slyke-Cullen constant and $K_{M}=K_{S}+K$ is known as the Michaelis-Menten constant. By introducing the following parameters

$$
\tau=\frac{k_{1}\left[E_{0}\right](t)}{\varepsilon}, u(\tau)=\frac{[S](t)}{\left[S_{0}\right]}, \mathrm{v}(\tau)=\frac{[C](t)}{\left[E_{0}\right]},
$$

$w(\tau)=\frac{[P](t)}{\left[E_{0}\right]}, \lambda=\frac{k_{2}}{k_{1}\left[S_{0}\right]}, k=\frac{K_{M}}{\left[S_{0}\right]}, \varepsilon=\frac{\left[E_{0}\right]}{\left[S_{0}\right]}$

the system of Eqs.2-4 with initial condition (5) can be represented in dimensionless form as follow:

$$
\begin{aligned}
& \frac{d u}{d \tau}=u \varepsilon+\varepsilon(u+k-\lambda) v \\
& \frac{d v}{d \tau}=u-(u+k) v \\
& \frac{d w}{d \tau}=\lambda v \\
& u(0)=1, v(0)=0, w(0)=0
\end{aligned}
$$

\section{ANALYTICAL SOLUTION OF STEADY STATE CONCENTRATION USING HOMOTOPY PERTURBATION ME- THOD}

Recently, many authors have applied the Homotopy perturbation method to various problems and demonstrated the efficiency of the Homotopy perturbation method for handling non-linear structures and solving various physics and engineering problems [15-18]. This method is a combination of topology and classic perturbation techniques. Ji Huan He used the Homotopy perturbation method to solve the Lighthill equation [19], the Duffing equation [20] and the Blasius equation [21]. The idea has been used to solve non-linear boundary value problems, integral equations and many other problems. In these papers [22-27], the homotopy perturbation method is applied and the obtained results show that the Homotopy perturbation method is very effective and simple. The Homotopy perturbation method is unique in its applicability, accuracy, efficiency and uses the imbedding parameter $p$ as a small parameter and only a few iterations are needed to search for an asymptotic solution. Using this method, we can obtain the following solution to Eqs.6-8 (Ref Appendix-A and B)

$$
\begin{aligned}
u(\tau) & =e^{-\varepsilon \tau}+\frac{e^{-\varepsilon \tau}}{k}+\frac{\varepsilon}{k-\varepsilon}\left[\frac{e^{-(\mathrm{k}+\varepsilon) \tau}}{k}-\frac{e^{-2 \varepsilon \tau}}{\varepsilon}\right] \\
& +\frac{\varepsilon(k-\lambda) \tau e^{-\varepsilon \tau}}{k-\varepsilon}+\frac{\varepsilon(k-\lambda)\left[e^{-\mathrm{k} \tau}-e^{-\varepsilon \tau}\right]}{(k-\varepsilon)^{2}} \\
v(\tau) & =\frac{e^{-\varepsilon \tau}-e^{-\mathrm{k} \tau}}{k-\varepsilon}+\frac{e^{-\mathrm{k} \tau}}{\varepsilon(-2 \varepsilon)} \\
& -\frac{e^{-2 \varepsilon \tau}}{(k-\varepsilon)(k-2 \varepsilon)}-\frac{e^{-(\mathrm{k}+\varepsilon) \tau}}{\varepsilon(k-\varepsilon)}
\end{aligned}
$$




$$
\begin{aligned}
w(\tau) & =\frac{\lambda\left(1-e^{-\varepsilon \tau}\right)}{\varepsilon(k-\varepsilon)}-\frac{\lambda\left(1-e^{-\mathrm{k} \tau}\right)}{\varepsilon(k-\varepsilon)}+\frac{\lambda\left(1-e^{-\varepsilon \tau}\right)}{k \varepsilon(k-2 \varepsilon)} \\
& -\frac{\lambda\left(1-e^{-2 \varepsilon \tau}\right)}{2 \varepsilon(k-\varepsilon)(k-2 \varepsilon)}-\frac{\lambda\left(1-e^{-(\mathrm{k}+\varepsilon) \tau}\right)}{\varepsilon(k+\varepsilon)(k-\varepsilon)}
\end{aligned}
$$

Eqs.10-12 represents the analytical expression of the dimensionless substrate concentration $u(\tau)$, enzyme substrate concentration $v(\tau)$ and product concentration $w(\tau)$ for all values of parameters $k, \lambda$ and $\varepsilon$.

\section{NUMERICAL SIMULATION}

The non-linear differential Eqs.2-4, are also solved using numerical methods. The functionbvp $4 \mathrm{c}$ in Scilab software which is a function of solving two-point boundary value problems (BVPs) for ordinary differential equations is used to solve this equation. Its numerical solution is compared with Homotopy perturbation method and it gives satisfactory result. The Scilab program is also given in Appendix (C).

\section{RESULT AND DISCUSSION}

Figures 1-6 show the analytical expression of conentrations of substrate $u$, enzyme-substrate complex $v$ and product $w$ for various values of dimensionless reaction parameters $k, \lambda$ and $\varepsilon$, wherein $k$ and $\lambda$ values are same and $\varepsilon$ is different. From these figures, it is inferred that the vlaue of the concentration of substrate decreases gradually from its intial value $(u(0)=1)$. The concentration of the substrate becomes

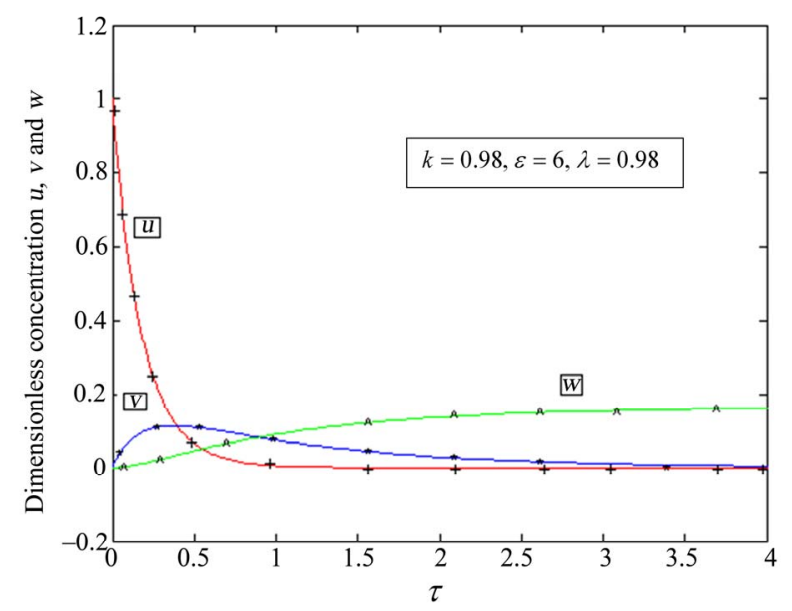

Figure 1. Normalised concentration profiles $u(\tau), v(\tau)$ and $w(\tau)$ as a function of dimensionless time for various values of reaction/diffusion parameter $k=0.98, \varepsilon=6$ and $\lambda=0.98$. These concentrations were computed using Eqs.10-12. The line denotes Eqs.10-12 and $+,{ }^{*}, \wedge$ denote the numerical simulation.

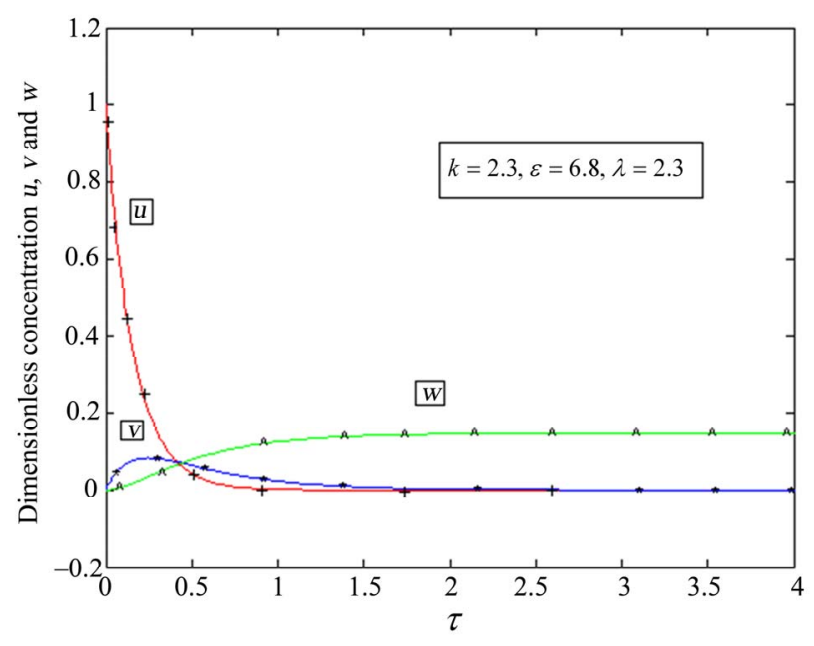

Figure 2. Normalised concentration profiles $u(\tau), v(\tau)$ and $w(\tau)$ as a function of dimensionless time for various values of reaction/diffusion parameter $k=2.3, \varepsilon=6.8$ and $\lambda=2.3$. These concentrations were computed using Eqs. 10-12. The line denotes Eqs.10-12 and $+, *, \wedge$ denote the numerical simulation.

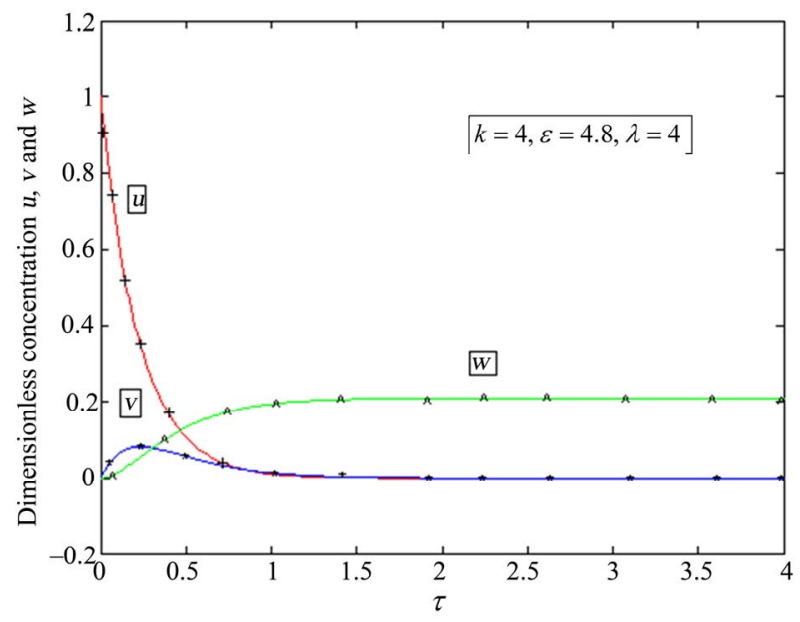

Figure 3. Normalised concentration profiles $u(\tau), v(\tau)$ and $w(\tau)$ as a function of dimensionless time for various values of reaction/diffusion parameter $k=4, \varepsilon=4.8$ and $\lambda=4$. These concentrations were computed using Eqs.10-12. The line denotes Eqs.10-12 and +, *, ^ denote the numerical simulation.

zero when $\tau \geq 0.5$ and reaches the steady-state value $(u=0)$ when $\tau \geq 1$. The enzyme substrate concentration $v$ increase gradually from its intial value $(v(0)=0)$ and attains maximum in the interval $[0,0.5]$ and degrees gradually, after that attains steady state value when $\tau \geq 1$ for all values of reaction parameter.

The concentration of the product $w$ increases slowly from the initial concentration $(w(0)=0)$, attains maximum value in the interval $[0.5,1]$ and attains steady state 


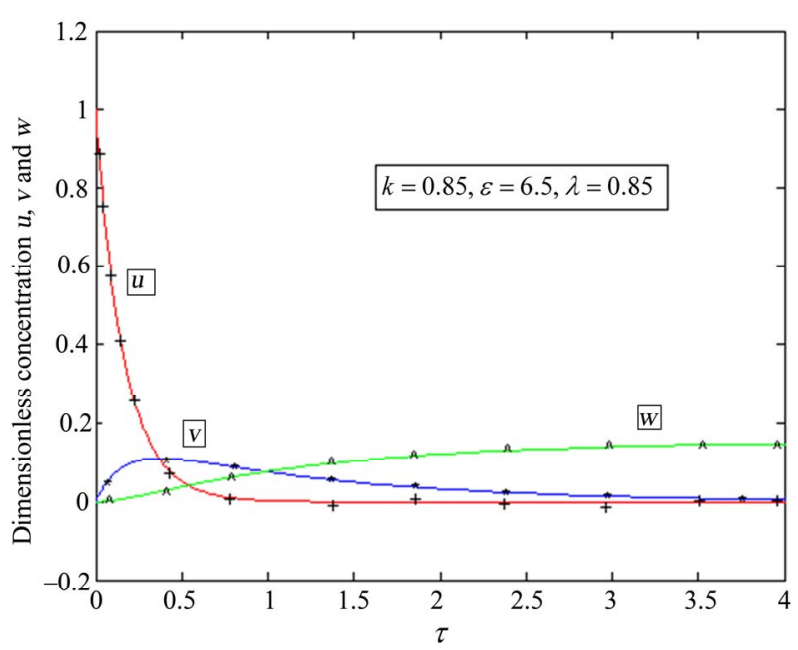

Figure 4. Normalised concentration profiles $u(\tau), v(\tau)$ and $w(\tau)$ as a function of dimensionless time for various values of reaction/diffusion parameter $k=0.85, \varepsilon=6.5$ and $\lambda=0.85$. These concentrations were computed using Eqs. 10-12. The line denotes Eqs.10-12 and $+, *, \wedge$ denote the numerical simulation.

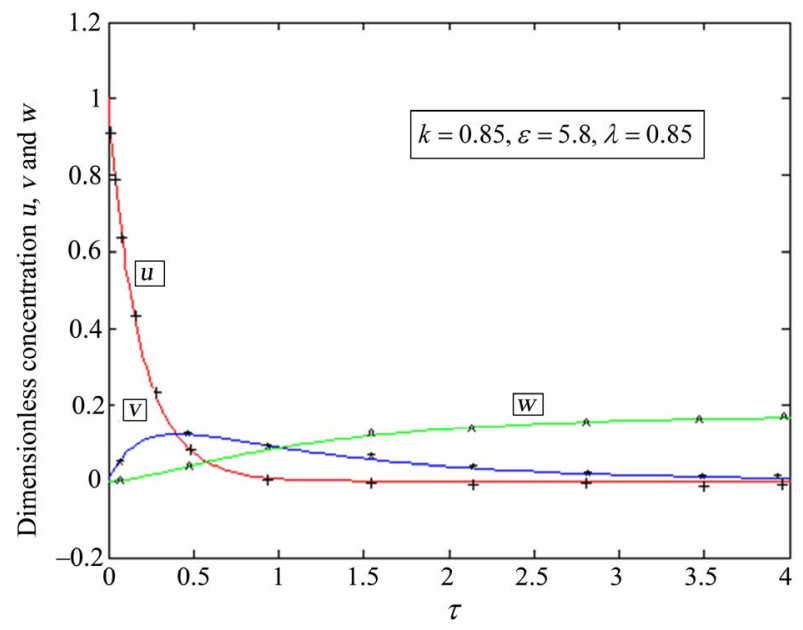

Figure 5. Normalised concentration profiles $u(\tau), v(\tau)$ and $w(\tau)$ as a function of dimensionless time for various values of reaction/diffusion parameter $k=0.98, \varepsilon=5.8$ and $\lambda=0.98$. These concentrations were computed using Eqs. 10-12. The line denotes Eqs.10-12 and $+,{ }^{*}, \wedge$ denote the numerical simulation.

when $\tau \geq 1$ for all values of reaction parameter. Also when the value of the parameter $\varepsilon$ decreases the product conentration increases. It is noted that in the interval $[1,1.5]$ concentration of substrate $u$ attains minimum value where as the product concentration attains its maximum value. Our approximate analytical expression of substrate concentration, enzyme substrate concentration and product concentration are compared with simulation results in Figures 1-6. A satisfactory agreement

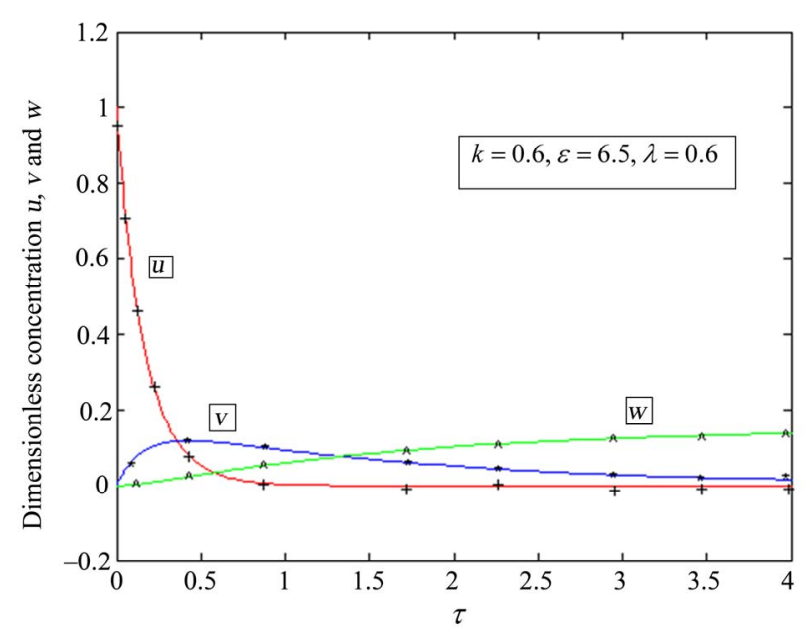

Figure 6. Normalised concentration profiles $u(\tau), v(\tau)$ and $w(\tau)$ as a function of dimensionless time for various values of reaction/diffusion parameter $k=0.6, \varepsilon=6.5$ and $\lambda=0.6$. These concentrations were computed using Eqs. 10-12. The line denotes Eqs.10-12 and $+,{ }^{*}, \wedge$ denote the numerical simulation.

is noted.

\section{CONCLUSIONS}

Pseudo-first-order kinetics serves the purpose of solving the set of differential equations governing the time course of the reaction, which can be validated by a proper choice of conditions. The time dependent nonlinear reaction-diffusion equation has been formulated and solved analytically and numerically. Analytical expressions for the substrate concentration, enzyme substrate concentration and product concentration have been derived interms of dimensionless reaction diffusion parameters $k, \lambda$ and $\varepsilon$ using the HPM. The primary result of this work is simple approximate calculations of concentrations for all values of dimensionless parameters $k, \lambda$ and $\varepsilon$ The HPM is an extremely simple method and it is also a promising method to solve other non-linear equations. This method can be easily extended to all kinds of system of coupled non-linear equations in multi-substrate systems and networks of coupled enzyme reactions.

\section{ACKNOWLEDGEMENTS}

This work was supported by the Department of Science and Technology (DST) Government of India. The authors also thank Mr.M.S.Meenakshisundaram, Secretary, The Madura College Board, Principal and S.Thiagarajan Head of the Department of Mathematics, The Madura College, Madurai, for their constant encouragement. It is our pleasure to thank the referees for their valuable comments. 


\section{REFERENCES}

[1] Rubinow, S.I. (1975) Introduction to Mathematical Biology. Wiley, New York.

[2] Murray, J.D. (1989) Mathematical biology. Springer Verlag, Berlin.

[3] Segel, L.A. (1980) Mathematical models in molecular and cellular biology. Cambridge University Press, Cambridge.

[4] Roberts, D.V. (1977) Enzyme kinetics. Cambridge University Press, Cambridge.

[5] Kasserra, H.P. and Laidler, K.J. (1970) Transient-phase studies of a trypsin-catalyzed reaction. Canadian Journal of Chemistry, 48, 1793-1802. doi:10.1139/v70-298

[6] Pettersson, G. (1976) The transient-state kinetics of two-substrate enzyme systems operating by an ordered ternary-complex mechanism. European Journal of Biochemistry, 69, 273-278. doi:10.1111/j.1432-1033.1976.tb10883.x

[7] Pettersson, G. (1978) A generalized theoretical treatment of the transient-state kinetics of enzymic reaction systems far from equilibrium. Acta Chemica Scandinavica Series B, 32, 437-446. doi:10.3891/acta.chem.scand.32b-0437

[8] Gutfreund, H. (1995) Kinetics for life sciences: Receptors, transmitters and catalysis. Cambridge University Press, Cambridge. doi:10.1017/CBO9780511626203

[9] Fersht, A.R. (1999) Structure and mechanism in protein science: A guide to enzyme catalysis and protein folding. Freeman, New York.

[10] Silicio, F. and Peterson, M.D. (1961) Ratio errors in pseudo first order reactions. Journal of Chemical Education, 38, 576-577. doi:10.1021/ed038p576

[11] Moore, J.W. and Pearson, R.G. (1981) Kinetics and Mechanism. Wiley, New York.

[12] Corbett, J.F. (1972) Pseudo first-order kinetics. Journal of Chemical Education, 49, 663. doi:10.1021/ed049p663

[13] Schnell, S. and Maini, P.K. (2000) Enzyme kinetics at high enzyme concentration. Bulletin of Mathematical Biology, 62, 483-499. doi:10.1006/bulm.1999.0163

[14] Schnell, S. and Mendoza, C. (2004) The condition for pseudo-first-order kinetics in enzymatic reaction is independent of the initial enzyme concentration. Journal of Biophysical Chemistry, 107, 165-174. doi:10.1016/j.bpc.2003.09.003

[15] Meena, A., Eswari, A. and Rajendran, L. (2010) Mathematical modelling of enzyme kinetics reaction mechanism and analytical sloutions of non-linear reaction equa- tions. Journal of Mathematical Chemistry, 48, 179-186. doi:10.1007/s10910-009-9659-5

[16] Li, S.J. and Liu, Y.X. (2006) An improved approach to nonlinear dynamical system identification using pid neural networks. International Journal of Nonlinear Science and Numerical Simulation, 7, 177-182.

[17] Mousa, M.M., Ragab, S.F. and Nturforsch, Z. (2008) Application of the homotopy perturbation method to linear and nonlinear schrödinger equations. Zeitschrift für Naturforschung, 63, 140-144.

[18] He, J.H. (1999) Homotopy perturbation technique. Computer Methods in Applied Mechanics and Engineering, 178, 257-262.

[19] He, J.H. (2003) Homotopy perturbation method: a new nonlinear analytical Technique. Applied Mathematics and Computation, 135, 73-79. doi:10.1016/S0096-3003(01)00312-5

[20] He, J.H. (2003) A Simple perturbation approach to Blasius equation. Applied Mathematics and Computation, 140, 217-222. doi:10.1016/S0096-3003(02)00189-3

[21] He, J.H. (2006) Some asymptotic methods for strongly nonlinear equations. International Journal of Modern Physics B, 20, 1141-1199. doi: $10.1142 / \mathrm{S} 0217979206033796$

[22] He, J.H., Wu, C.G. and Austin, F. (2010) The variational iteration method which should be followed. Nonlinear Science Letters A, 1, 1-30.

[23] He, J.H. (2003) A coupling method of a homotopy technique and a perturbation technique for non-linear problems. International Journal of Non-Linear Mechanics, 35, 37-43. doi:10.1016/S0020-7462(98)00085-7

[24] Ganji, D.D., Amini, M. and Kolahdooz, A. (2008) Analytical investigation of hyperbolic equations via he's methods. American Journal of Engineering and Applied Sciences, 1, 399-407.

[25] Loghambal, S. and Rajendran, L. (2010) Mathematical modeling of diffusion and kinetics of amperometric immobilized enzyme electrodes. Electrochimica Acta, 55, 5230-5238. doi:10.1016/j.electacta.2010.04.050

[26] Meena, A. and Rajendran, L. (2010) Mathematical modeling of amperometric and potentiometric biosensors and system of non-linear equations-Homotopy perturbation approach. Journal of Electroanalytical Chemistry, 644, 50-59. doi:10.1016/j.jelechem.2010.03.027

[27] Eswari, A. and Rajendran, L. (2010) Analytical solution of steady state current an enzyme modified microcylinder electrodes. Journal of Electroanalytical Chemistry, 648, 36-46. doi:10.1016/j.jelechem.2010.07.002 


\section{APPENDIX A}

\section{Basic idea of Homotopy - perturbation me- thod (HPM)}

To explain this method, let us consider the following function

$$
A(w)-f(r)=0, r \in \Omega
$$

With the boundary conditions of

$$
B\left(w, \frac{\partial w}{\partial n}\right)=0, r \in \Gamma
$$

where $A, B, f(r)$ and $\Gamma$ are a general differential operator, a boundary operator, a known analytical function and the boundary of the domain $\Omega$, respectively. Generally speaking, the operator $A$ can be divided into a linear part $L$ and a nonlinear part $N$. Eq.A1 can therefore, be written as

$$
L(w)+N(w)-f(r)=0
$$

By the Homotopy technique, we construct a Homotopy $z(r, p): \Omega \times[0,1] \rightarrow R$ which satisfies

$$
\begin{aligned}
H(z, p) & =(1-p)\left[L(z)-L\left(w_{0}\right)\right] \\
& +p(A(z)-f(r))=0 \quad p \in[0,1], r \in \Omega
\end{aligned}
$$

Or

$$
H(z, p)=L(z)-L\left(w_{0}\right)+p L\left(w_{0}\right)+p[N(z)-f(r)]=0
$$

where $p \in[0,1]$ is an embedding parameter, while $w_{0}$ is an initial approximation of Eq.A1, which satisfies the boundary conditions. Obviously, from Eqs.A4 and A5, we will have

$$
\begin{aligned}
& H(z, 0)=L(z)-L\left(w_{0}\right)=0 \\
& H(z, 1)=A(z)-f(r)=0 .
\end{aligned}
$$

The changing process of $p$ from zero to unity is just that of $z(r, p)$ from $w_{0}$ to $w(r)$. In topology, this is called deformation, while $L(z)-L\left(w_{0}\right)$ and $A(z)-f(r)$ are called Homotopy. According to the HPM, we can first use the embedding parameter $p$ as a "small parameter", and assume that the solutions of Eqs. A4 and A5 can be written as a power series in $p$ :

$$
z=z_{0}+p z_{1}+p^{2} z_{2}+\ldots . .
$$

Setting $p=1$ results in the approximate solution of Eq. A1

$$
w=\lim _{p \rightarrow 1} z=z_{0}+z_{1}+z_{2}+\ldots .
$$

The combination of the perturbation method and the Homotopy method is called the HPM, which eliminates the drawbacks of the traditional perturbation methods while keeping all its advantages.

\section{APPENDIX B}

Solution of the Eqs. 2 and $\mathbf{3}$ using Homotopy perturbation method. In this Appendix, we indicate how Eqs.10, $\mathbf{1 1}$ and $\mathbf{1 2}$ in this paper are derived. Furthermore, a Homotopy was constructed to determine the solution of Eqs.2 and 3.

$$
\begin{aligned}
&(1-p)\left[\frac{d u}{d \tau}+u\right.\varepsilon] \\
&+ p\left[\frac{d u}{d \tau}+u \varepsilon-\varepsilon u v-\varepsilon k v+\lambda \varepsilon v\right]=0 \\
&(1-p)\left[\frac{d v}{d \tau}+k v\right]+p\left[\frac{d v}{d \tau}+k v-u+u v\right]=0
\end{aligned}
$$

and the initial approximations are as follows:

$$
u(0)=1, v(0)=0
$$

approximate solutions of (B1) and (B2) are

$$
u=u_{0}+p u_{1}+p^{2} u_{2}+p^{3} u_{3}+
$$

and

$$
v=v_{0}+p v_{1}+p^{2} v_{2}+p^{3} v_{3}+
$$

Substituting Eqs.B4 and B5 into Eqs.B1 and B2 and comparing the coefficients of like powers of $p$

$$
\begin{gathered}
p^{0}: \frac{d u_{0}}{d \tau}+\varepsilon u_{0}=0 \\
p^{1}: \frac{d u_{1}}{d \tau}+\varepsilon u_{1}-\varepsilon u_{0} v_{0}-\varepsilon k v_{0}+\lambda \varepsilon v_{0}=0 \\
p^{2}: \frac{d u_{2}}{d \tau}+\varepsilon u_{2}-\varepsilon u_{0} v_{1}-\varepsilon u_{1} v_{0}-\varepsilon k v_{1}+\lambda \varepsilon v_{1}=0 \\
\text { And } \quad p^{0}: \frac{d v_{0}}{d \tau}+k v_{0}=0 \\
p^{1}: \frac{d v_{1}}{d \tau}+k v_{1}-u_{0}+u_{0} v_{0}=0 \\
p^{2}: \frac{d v_{2}}{d \tau}+k v_{2}-u_{1}+u_{0} v_{1}+u_{1} v_{0}=0
\end{gathered}
$$

And

Solving the Eqs.B6-B11, and using the boundary conditions (B3), we can find the following results

$$
\begin{aligned}
& u_{0}(\tau)=e^{-\varepsilon \tau} \\
& u_{1}(\tau)=0
\end{aligned}
$$

$$
u_{2}(\tau)=\frac{e^{-\varepsilon \tau}}{k}+\frac{\varepsilon}{k-\varepsilon}\left[\frac{e^{-(\mathrm{k}+\varepsilon) \tau}}{k}-\frac{e^{-2 \varepsilon \tau}}{\varepsilon}\right]
$$


$+\frac{\varepsilon(k-\lambda) \tau e^{-\varepsilon \tau}}{k-\varepsilon}+\frac{\varepsilon(k-\lambda)\left[e^{-\mathrm{k} \tau}-e^{-\varepsilon \tau}\right]}{(k-\varepsilon)^{2}}$

And

$$
\begin{gathered}
v_{0}(\tau)=0 \\
v_{1}(\tau)=\frac{e^{-\varepsilon \tau}-e^{-\mathrm{k} \tau}}{k-\varepsilon} \\
v_{2}(\tau)=\frac{e^{-\mathrm{k} \tau}}{\varepsilon(k-2 \varepsilon)}-\frac{e^{-2 \varepsilon \tau}}{(k-\varepsilon)(k-2 \varepsilon)}-\frac{e^{-(\mathrm{k}+\varepsilon) \tau}}{\varepsilon(k-\varepsilon)}
\end{gathered}
$$

According to the HPM, we can conclude that

$$
\begin{aligned}
& u(\tau)=\lim _{p \rightarrow 1} u(\tau)=u_{0}+u_{1}+u_{2} \\
& v(\tau)=\lim _{p \rightarrow 1} v(\tau)=v_{0}+v_{1}+v_{2}
\end{aligned}
$$

Using Eqs.B12, B13 and B14 in Eqs.B18 and Eqs. B15, B16 and B17 in Eqs.B19, we obtain the final results as described in Eqs.10 and 11. The dimensionless concentration of the product is given by

$$
\begin{aligned}
& w(\tau)=\lambda \int_{0}^{\tau} \mathrm{v}(\tau) d \tau \\
& =\frac{\lambda\left(1-e^{-\varepsilon \tau}\right)}{\varepsilon(\mathrm{k}-\varepsilon)}-\frac{\lambda\left(1-e^{-\mathrm{k} \tau}\right)}{\varepsilon(\mathrm{k}-\varepsilon)}+\frac{\lambda\left(1-e^{-\varepsilon \tau}\right)}{\mathrm{k} \varepsilon(\mathrm{k}-2 \varepsilon)} \\
& -\frac{\lambda\left(1-e^{-2 \varepsilon \tau}\right)}{2 \varepsilon(\mathrm{k}-\varepsilon)(\mathrm{k}-2 \varepsilon)}-\frac{\lambda\left(1-e^{-(\mathrm{k}+\varepsilon) \tau}\right)}{\varepsilon(\mathrm{k}+\varepsilon)(\mathrm{k}-\varepsilon)}
\end{aligned}
$$

The above equation represent the new analytical expression of product $w(\tau)$ for all values of parameters $k, \lambda$ and $\varepsilon$ which is given in Eq.12.

\section{APPENDIX C}

Scilab program to find the solutions of the Eqs. 6-9 function main 123456

options= odeset('RelTol',1e-6,'Stats','on');

$\%$ initial conditions

$\mathrm{x} 0=[1 ; 0 ; 0]$;

$\operatorname{tspan}=\left[\begin{array}{ll}0 & 10\end{array}\right]$;

tic

$[\mathrm{t}, \mathrm{x}]=$ ode45(@TestFunction,tspan,x0,options); toc

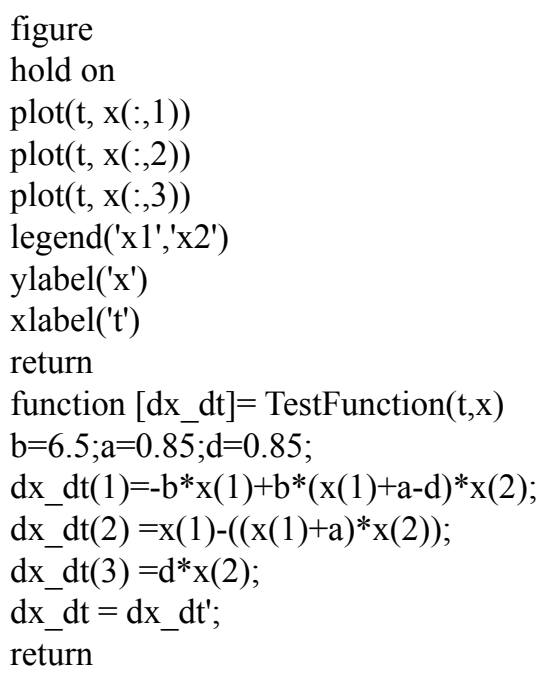

\section{APPENDIX D}

\section{Nomenclature}

\section{Symboles}

$[E]$

$[C]$

$[S]$

$\left[E_{0}\right]$

$\left[S_{0}\right]$

$K_{M}$

$K_{S}$

$(\mu \mathrm{M})$

K

$k_{1}, k_{-1}, k_{2}$

Enzyme concentration ( $\mu \mathrm{M}$ )

$k, \lambda, \varepsilon$

Enzyme-substrate complex $\quad(\mu \mathrm{M})$

Substrate concentation $(\mu \mathrm{M})$

Initial enzyme concentration $(\mu \mathrm{M})$

Initial substrate concentraton $(\mu \mathrm{M})$

Michaelis-Menten constant

Equilibrium dissociation constant

$u$ tion (None)

$v$ centration (None)

$w$

(None)

$t$

$\tau$
Van Slyke-Cullen constant ( $\mu \mathrm{M}$ )

Positive rate constants (None)

Reaction diffusion parameter (None)

Dimensionless Substrate concentra-

Dimensionless enzyme substrate con-

Dimensionless product concentration

Time (Sec)

Dimensionless time (None) 